\title{
Long-time behavior of solutions for a fractional diffusion problem
}

Ailing $\mathrm{Qi}^{1}$, Die $\mathrm{Hu}^{1}$ and Mingqi Xiang ${ }^{1 *}$ (D)

\section{"Correspondence:}

xiangmingqi_hit@163.com

${ }^{1}$ College of Science, Civil Aviation University of China, Tianjin, 300300, P.R. China

\section{Springer}

\section{Abstract}

This paper deals with the asymptotic behavior of solutions to the initial-boundary value problem of the following fractional $p$-Kirchhoff equation:

$$
u_{t}+M\left([u]_{s, p}^{p}\right)(-\Delta)_{p}^{s} u+f(x, u)=g(x) \text { in } \Omega \times(0, \infty),
$$

where $\Omega \subset \mathbb{R}^{N}$ is a bounded domain with Lipschitz boundary, $N>p s, 0<s<1<p$, $M:[0, \infty) \rightarrow[0, \infty)$ is a nondecreasing continuous function, $[u]_{s, p}$ is the Gagliardo seminorm of $u, f: \Omega \times \mathbb{R} \rightarrow \mathbb{R}$ and $g \in L^{2}(\Omega)$. With general assumptions on $f$ and $g$, we prove the existence of global attractors in proper spaces. Then, we show that the fractal dimensional of global attractors is infinite provided some conditions are satisfied.

MSC: $35 \mathrm{~K} 55 ; 35 \mathrm{R} 11 ; 47 \mathrm{G} 20$

Keywords: Fractional p-Laplacian; Global attractors; Fractal dimension

\section{Introduction and main results}

Let $s \in(0,1)$ and let $\Omega$ be a bounded domain in $\mathbb{R}^{N}(N>2 s)$ with smooth boundary $\partial \Omega$. We consider the asymptotic behavior of solutions to the following fractional $p$-Kirchhoff problem:

$$
\begin{cases}u_{t}+M\left([u]_{s, p}^{p}\right)(-\Delta)_{p}^{s} u+f(x, u)=g(x) & \text { in } \Omega \times \mathbb{R}^{+}, \\ u=0 & \text { on }\left(\mathbb{R}^{N} \backslash \Omega\right) \times \mathbb{R}^{+}, \\ u(x, 0)=u_{0}(x) & \text { in } \Omega,\end{cases}
$$

where $u_{0} \in L^{2}(\Omega), g \in L^{2}(\Omega),[u]_{s, p}^{p}=\iint_{\mathcal{Q}} \frac{\mid u(x, t)-u\left(y,\left.t\right|^{\mid}\right.}{\left.|x-y|\right|^{N+p s}} d x d y$ with $\mathcal{Q}=\mathbb{R}^{2 N} \backslash(\mathcal{C} \Omega \times \mathcal{C} \Omega)$ and $\mathcal{C} \Omega=\mathbb{R}^{N} \backslash \Omega, M: \mathbb{R}_{0}^{+} \rightarrow \mathbb{R}_{0}^{+}$is a continuous function, and $(-\Delta)_{p}^{s}$ is the fractional $p$-Laplacian, which (up to normalization factors) may be defined by

$$
(-\Delta)_{p}^{s} \varphi(x)=2 \lim _{R \rightarrow 0^{+}} \int_{\mathbb{R}^{N} \backslash B_{R}(x)} \frac{|\varphi(x)-\varphi(y)|^{p-2}(\varphi(x)-\varphi(y))}{|x-y|^{N+p s}} d y
$$

(c) The Author(s) 2021. This article is licensed under a Creative Commons Attribution 4.0 International License, which permits use, sharing, adaptation, distribution and reproduction in any medium or format, as long as you give appropriate credit to the original author(s) and the source, provide a link to the Creative Commons licence, and indicate if changes were made. The images or other third party material in this article are included in the article's Creative Commons licence, unless indicated otherwise in a credit line to the material. If material is not included in the article's Creative Commons licence and your intended use is not permitted by statutory regulation or exceeds the permitted use, you will need to obtain permission directly from the copyright holder. To view a copy of this licence, visit http://creativecommons.org/licenses/by/4.0/. 
for all $\varphi \in C_{0}^{\infty}\left(\mathbb{R}^{N}\right)$, where $1<p<\frac{N}{s}$ and $s \in(0,1)$. Henceforward $B_{R}(x)$ denotes the ball of $\mathbb{R}^{N}$ centered at $x \in \mathbb{R}^{N}$ and radius $R>0$. We assume that $f: \Omega \times \mathbb{R} \rightarrow \mathbb{R}$ is a Carathéodory function satisfying:

$\left(f_{1}\right)$ there exists a positive constant $\lambda$ such that

$$
\left(f\left(x, \xi_{1}\right)-f\left(x, \xi_{2}\right)\right)\left(\xi_{1}-\xi_{2}\right) \geq-\lambda\left|\xi_{1}-\xi_{2}\right|^{2}
$$

for any $(x, t) \in \Omega \times \mathbb{R}^{+}$and $\xi_{1}, \xi_{2} \in \mathbb{R}$;

$\left(f_{2}\right)$ there exist positive constants $c, c_{1}, c_{2}$ such that

$$
c_{1}|\xi|^{q}-c \leq f(x, \xi) \xi \leq c_{2}|\xi|^{q}+c \quad \text { for any }(x, t) \in \Omega \times \mathbb{R}^{+} \text {and } \xi \in \mathbb{R}
$$

where $q$ satisfies $2 \leq q<\infty$.

A typical example of $f$ is given by $f(x, \xi)=a(x)|\xi|^{q-2} \xi-b(x)|\xi|^{r-2} \xi$ for all $x \in \Omega$ and $\xi \in \mathbb{R}$, with $2<r<q<\infty$. Here $a, b$ are two bounded continuous functions in $\Omega$.

Throughout this paper, we always assume that $s \in(0,1), N>2 s, u_{0}(x) \in L^{2}(\Omega) \cap W_{0}$ is a nonzero function. For the coefficient function $M$, we assume that

$\left(M_{1}\right) M:[0, \infty) \rightarrow(0, \infty)$ is a nondecreasing and continuous function and there exists $m_{0}>0$ such that $M(\tau) \geq m_{0}$ for all $\tau \geq 0$.

In particular, if $p=2$, then $(-\Delta)_{p}^{s}$ reduces to the fractional Laplace operator $(-\Delta)^{s}$, see [5] and the references cited there. For the basic properties of fractional $p$-Laplacian, we refer the readers to $[13,16,17]$. Problem $(1.1)$ is a class of nonlocal fractional diffusion problems. It is relevant in anomalous diffusion theory. More precisely, if $p=2$ and $M \equiv 1$, as stated in [7], the function $u(x, t)$ is thought of as a density of population at the point $x$ and time $t$, the term $(-\Delta)^{s} u(x, t)$ represents the diffusion of density, $f(x, u)$ is the source term, $g$ is the perturbation term, and the coefficient $M:[0, \infty) \rightarrow(0, \infty)$ denotes the possible changes of total population in whole space. This implies that the behavior of individuals is subject to total population, such as the diffusion process of bacteria, see [21, 27]. It is worth mentioning that the interest in fractional problems goes beyond the mathematical curiosity. Actually, the study of problems like (1.1) has many significant applications, as explained by Caffarelli in [2, 3], Laskin in [15], and Vázquez in [31]. Very recently, Fiscella and Valdinoci [9] first proposed a stationary Kirchhoff variational equation which models the nonlocal aspect of the tension of the string. Indeed, the stationary problem (1.1) is a fractional version of a model, the so-called stationary Kirchhoff equation, introduced by Kirchhoff in [14]. The body of literature on elliptic type problems involving nonlocal operators is quite large, here we just collect a few works, see [1, 8, 9, 19-22, 26, 28, 33, 36] and the references cited there.

To the best of our knowledge, there are a few papers that deal with the asymptotic behavior of solutions for problems like (1.1). Pucci, Xiang, and Zhang [27] discussed the initial-boundary value problem of the following equation:

$$
u_{t}+M\left([u]_{s, p}^{p}\right)(-\Delta)_{p}^{s} u=f(x, t) \text { in } \Omega \times(0, \infty)
$$

where $M:[0, \infty) \rightarrow(0, \infty)$ is a continuous and nondecreasing function. Using the subdifferential approach, the authors obtained the well-posedness of solutions for (1.4). Moreover, the large-time behavior and extinction property of solutions were given. In [25], Pan, 
Zhang, and Cao studied the initial-boundary value problem of the following fractional $p$ Kirchhoff equation:

$$
u_{t}+[u]_{s, p}^{(\lambda-1) p}(-\Delta)_{p}^{s} u=|u|^{q-2} u \quad \text { in } \Omega \times(0, \infty)
$$

where $1 \leq \lambda<N /(N-s p)$ and $p<q<N p /(N-s p)$ with $1<p<N / s$. The existence of a global solution was obtained by employing the Galerkin method and the potential well theory. Xiang, Rădulescu, and Zhang [21] studied the initial-boundary value problem of the following fractional Kirchhoff equation:

$$
u_{t}+M\left([u]_{s, 2}^{2}\right)(-\Delta)^{s} u=|u|^{p-2} u \quad \text { in } \Omega \times(0, \infty)
$$

where $M:[0, \infty) \rightarrow(0, \infty)$ is continuous, and there exist $m_{0}>0$ and $\theta>1$ such that $M(\sigma) \geq m_{0} \sigma^{\theta-1}$ for all $\sigma \geq 0$, and $M$ also satisfies the following:

$(M)$ There exists $\theta \in\left[1, p_{s}^{*} / p\right)$ such that $t M(t) \leq \theta \mathscr{M}(t)$ for all $t \geq 0$, where $\mathscr{M}(t)=$ $\int_{0}^{t} M(\tau) d \tau$.

Under suitable assumptions, the authors obtained the local existence of nonnegative solutions by applying the Galerkin method and proved that the local nonnegative solutions blow up in finite time. In [34], Xiang and Yang studied the initial-boundary value of the following equation:

$$
u_{t}+M\left([u]_{s, p}^{p}\right)(-\Delta)_{p}^{s} u=\lambda|u|^{q-2} u-\mu|u|^{r-2} u .
$$

The authors gave some sufficient conditions such that the solutions of the above equation vanish in finite time. Moreover, the non-extinction of solutions was also investigated.

Very recently, Wang and Huang [32] considered a weakly damped fractional nonlinear Schrödinger equation on the real line $\mathbb{R}$

$$
u_{t}-i(-\Delta) u^{s}+i|u|^{2} u+\gamma u=f(x), \quad u(x, 0)=u_{0}(x)
$$

where $s \in(1 / 2,1), \gamma>0$, and $f \in L^{2}(\mathbb{R})$. The authors proved that (1.5) possesses a finite dimensionality of global attractor. In [12], Hurtado studied the following initial-boundary value problem of fractional Laplacian equation with variable exponents:

$$
u_{t}+(-\Delta)_{p(\cdot)}^{s} u=f(x, u)
$$

where $(-\Delta)_{p(\cdot)}^{s}$ is the fractional $p(\cdot)$-Laplacian defined as

$$
(-\Delta)_{p}^{s} u(x)=2 \lim _{R \rightarrow 0^{+}} \int_{\mathbb{R}^{N} \backslash B_{R}(x)} \frac{|u(x)-u(y)|^{p(x, y)-2}(u(x)-u(y))}{|x-y|^{N+p(x, y) s}} d y,
$$

and $p \in C(\bar{\Omega} \times \bar{\Omega})$. The author established the well-posedness of solutions by using the techniques of monotone operators. Moreover, the author obtained the existence of global attractors under suitable assumptions.

When $s=1$ and $M \equiv 1$, the equation in (1.1) reduces to the following equation:

$$
u_{t}-\Delta_{p} u+f(x, u)=g(x) \quad \text { in } \Omega
$$


where $\Delta_{p} u=\operatorname{div}\left(|\nabla u|^{p-2} \nabla u\right)$ is the $p$-Laplacian. In [37], Yang et al. obtained the existence of global attractors for (1.7). Indeed, the existence of global attractors is an important asymptotic property of solutions for parabolic equations which have been studied extensively by many researchers, see for example $[6,10,23,24,38]$.

In this paper, inspired by the above-mentioned papers, we discuss the existence of global attractors and fractal dimension of solutions for problem (1.1) involving the fractional $p$-Laplacian and nonlocal diffusion coefficient. Motivated by [23, 37], we first study the existence and uniqueness of solutions by using the Galerkin method. Then we give the existence of global attractors in proper spaces and obtain the fractal dimension of the global attractors. Since problem (1.1) contains a nonlocal coefficient $M$, in order to get the uniqueness of solutions, we impose a monotonicity assumption on $M$. However, to the best of our knowledge, there are no results on the existence of global attractors for problem (1.1) in the literature.

To introduce our main results, we first give the definition of (weak) solutions, see [25, 27].

Definition 1.1 For any fixed $T>0$, a function $u \in L^{p}\left(0, T ; W_{0}\right) \cap C\left(0, T ; L^{2}(\Omega)\right) \cap L^{q}(\Omega \times$ $(0, T))$ is said to be a (weak) solution of problem (1.1) if $u_{t} \in L^{2}\left(0, T ; L^{2}(\Omega)\right)$ and, for a.e. $t \in(0, T)$,

$$
\int_{\Omega} u_{t} \varphi d x+M\left([u]_{s, p}^{p}\right)\langle u, \varphi\rangle_{W_{0}}+\int_{\Omega} f(x, u) \varphi d x=\int_{\Omega} g(x) \varphi d x
$$

for all $\varphi \in W_{0}$, where

$$
\langle u, \varphi\rangle_{W_{0}}=\iint_{\mathcal{Q}} \frac{|u(x, t)-u(y, t)|^{p-2}[u(x, t)-u(y, t)][\varphi(x)-\varphi(y)]}{|x-y|^{N+s p}} d x d y,
$$

and $W_{0}$ is the fractional Sobolev space which will be introduced in Sect. 2.

Theorem 1.1 Assume that $2 \leq p<N / s, M$ satisfies $\left(M_{1}\right)$, $f$ fulfils $\left(f_{1}\right)-\left(f_{2}\right)$ with $2 \leq q<$ $N p /(N-s p)$, and $g \in L^{2}(\Omega)$. Then the semigroup associated with problem (1.1) admits a global attractor $\mathscr{A}_{q}$ in $L^{q}(\Omega)$,i.e., $\mathscr{A}_{q}$ is compact, invariant in $L^{q}(\Omega)$ and attracts every bounded subset of $L^{2}(\Omega)$ in the norm topology of $L^{q}(\Omega)$.

Remark 1.1 The monotonicity assumption on $M$ is just for getting the uniqueness of solutions.

Finally, we consider the following problem:

$$
\begin{cases}u_{t}+M\left([u]_{s, p}^{p}\right)(-\Delta)_{p}^{s} u+|u|^{q-2} u=|u|^{r-2} u & \text { in } \Omega \times \mathbb{R}^{+}, \\ u=0 & \text { on }\left(\mathbb{R}^{N} \backslash \Omega\right) \times \mathbb{R}^{+}, \\ u(x, 0)=u_{0}(x) & \text { in } \Omega .\end{cases}
$$

Theorem 1.2 Assume that $0<s<1,2<r<p<N / s, r<q<N p /(N-s p)$, and M satisfies $\left(M_{1}\right)$. Then the semigroup associated with problem (1.9) admits a symmetric global attractor in $L^{q}(\Omega)$ and its fractal dimension is infinite. 
The rest of the paper is organized as follows. In Sect. 2, we give some preliminary lemmas. The existence of global attractors is proved in Sect. 3. The existence of infinite dimensional attractors is obtained in Sect. 4.

\section{Preliminaries}

In this section, we first recall some necessary definitions and properties of the fractional Sobolev spaces, see $[5,8,9,13,35]$ for further details. From now on, we shortly denote by $\|\cdot\|_{v}$ the norm of $L^{v}(\Omega)(v \geq 1)$. Let $0<s<1<p<\infty$ be real numbers and the fractional critical exponent $p_{s}^{*}$ be defined as

$$
p_{s}^{*}= \begin{cases}\frac{N p}{N-s p}, & s p<N \\ \infty, & \text { otherwise }\end{cases}
$$

In the following, we set $\mathcal{Q}=\mathbb{R}^{2 N} \backslash(\mathcal{C} \Omega \times \mathcal{C} \Omega)$, where $\mathcal{C} \Omega=\mathbb{R}^{N} \backslash \Omega$.W is a linear space of Lebesgue measurable functions from $\mathbb{R}^{N}$ to $\mathbb{R}$ such that the restriction to $\Omega$ of any function $u$ in $W$ belongs to $L^{p}(\Omega)$ and

$$
\iint_{\mathcal{Q}} \frac{|u(x)-u(y)|^{p}}{|x-y|^{N+s p}} d x d y<\infty
$$

The space $W$ is equipped with the norm

$$
\|u\|_{W}=\|u\|_{L^{p}(\Omega)}+\left(\iint_{Q} \frac{|u(x)-u(y)|^{p}}{|x-y|^{N+s p}} d x d y\right)^{\frac{1}{p}} .
$$

It is easy to get that $\|\cdot\|_{W}$ is a norm on $W$. We shall work in the closed linear subspace

$$
W_{0}=\left\{u \in W: u(x)=0 \text { a.e. in } \mathbb{R}^{N} \backslash \Omega\right\} .
$$

By the result in [35], one can deduce that

$$
[u]_{s, p}=\left(\iint_{\mathcal{Q}} \frac{|u(x)-u(y)|^{p}}{|x-y|^{N+s p}} d x d y\right)^{\frac{1}{p}}
$$

is an equivalent norm of $W_{0}$.

Definition 2.1 (see $[23,24]$ ) Let $\{S(t)\}_{t \geq 0}$ be a semigroup on Banach space $X$. A subset $\mathscr{A} \subset X$ is called a global attractor for the semigroup if $\mathscr{A}$ is compact in $X$ and satisfies the following properties:

(1) $\mathscr{A}$ is invariant, i.e., $S(t) \mathscr{A}=\mathscr{A}$ for all $t \geq 0$;

(2) $\mathscr{A}$ attracts all bounded subset of $X$, that is, for any bounded subset $B \subset X$,

$$
\operatorname{dist}(S(t) B, \mathscr{A}) \rightarrow 0 \quad \text { as } t \rightarrow \infty,
$$

where $\operatorname{dist}(A, B)$ is the Hausdorff semidistance of two sets $A$ and $B$ given by

$$
\operatorname{dist}(A, B)=\sup _{x \in A} \inf _{y \in B}\|x-y\|_{X} .
$$


Definition 2.2 (see $[23,24]$ ) Let $\{S(t)\}_{t \geq 0}$ be a semigroup on Banach space $X$. A subset $B_{0} \subset X$ is called an absorbing set for the semigroup $\{S(t)\}_{t \geq 0}$ if $B_{0}$ satisfies that, for any bounded subset $B \subset X$, there exists a positive constant $T=T(B)$ such that

$$
S(t) B \subset B_{0} \quad \text { for any } t \geq T \text {. }
$$

Definition 2.3 (see [4]) Let $X$ be a metric space. Assume that $M$ is a compact subset in $X$. The fractal dimension $\operatorname{dim}_{f} M$ of $M$ is defined by

$$
\operatorname{dim}_{f} M=\limsup _{\varepsilon \rightarrow 0} \frac{\ln n(M, \varepsilon)}{\ln (1 / \varepsilon)}
$$

where $n(M, \varepsilon)$ denotes the minimal number of closed balls of the radius $\varepsilon$ which cover the set $M$.

\section{Global attractors in $L^{q}(\Omega)$}

In this section, we provide the existence results for problem (1.1), and then we show the existence of a global attractor in $L^{q}(\Omega)$.

Theorem 3.1 Assume that $2 \leq p<N / s, M$ satisfies $\left(M_{1}\right), g$ satisfies $\left(g_{1}\right)$, and $f$ fulfils $\left(f_{1}\right)$ $\left(f_{2}\right)$ with $2 \leq q<N p /(N-s p)$. Then problem (1.1) admits a unique solution

$$
u \in C\left([0, T] ; L^{2}(\Omega)\right) \cap L^{p}\left(0, T ; W_{0}\right) \cap L^{q}\left(0, T ; L^{q}(\Omega)\right) .
$$

Moreover, the mapping $u_{0} \rightarrow u(t)$ is continuous in $L^{2}(\Omega)$.

Proof The existence of solutions for problem (1.1) can be obtained by using the Galerkin method, see for example [21, 25]. For completeness, we give a sketch of the proof.

Choose a sequence of functions $\left\{e_{j}\right\}_{j=1}^{\infty} \subset C_{0}^{\infty}(\Omega)$ which is an orthonormal basis in $L^{2}(\Omega)$. We shall find the approximate solutions as follows:

$$
u_{n}(x, t)=\sum_{j=1}^{n}\left(\eta_{n}(t)\right)_{j} e_{j}(x) \quad \text { for all } n \in \mathbb{N},
$$

where the unknown functions $\left(\eta_{n}(t)\right)_{j}$ are determined by the following ODEs:

$$
\left\{\begin{array}{l}
\eta_{n}^{\prime}(t)=-I_{n}\left(t, \eta_{n}(t)\right), \quad t \in \mathbb{R}^{+}, \\
\eta_{n}(0)=U_{0 n} .
\end{array}\right.
$$

Here $U_{0 n}=\left(\int_{\Omega} u_{0 n}(x) e_{1}(x) d x, \ldots, \int_{\Omega} u_{0 n}(x) e_{n}(x) d x\right), u_{0 n} \rightarrow u_{0}$ in $W_{0}$,

$$
\begin{aligned}
\left(I_{n}\left(t, \eta_{n}\right)\right)_{j}= & M\left(\left[u_{n}\right]_{s, p}^{p}\right)\left\langle u_{n}, e_{j}\right\rangle_{W_{0}}+\int_{\Omega} f\left(x, u_{n}(x, t)\right) e_{j}(x) d x \\
& -\int_{\Omega} g(x) e_{j}(x) d x, \quad j=1,2, \ldots, n .
\end{aligned}
$$


The definition of $\left\langle u_{n}, e_{j}\right\rangle_{W_{0}}$ is given by

$$
\left\langle u_{n}, e_{j}\right\rangle_{W_{0}}=\iint_{\mathcal{Q}} \frac{\left|u_{n}(x, t)-u_{n}(y, t)\right|^{p-2}\left[u_{n}(x, t)-u_{n}(y, t)\right]\left[e_{j}(x)-e_{j}(y)\right]}{|x-y|^{N+s p}} d x d y .
$$

By the continuity of $M$ and the definition of $I_{n}$, we know that $I_{n}$ is continuous on $\mathbb{R}_{0}^{+} \times \mathbb{R}^{n}$. Then the Peano theorem (see [11]) yields that there exists a local solution of problem (3.1) on $\left(0, T_{n}\right)\left(0<T_{n}<\infty\right)$. The following a priori estimate implies that the local solution can be extended to $(0, \infty)$.

Multiplying (3.1) by $\eta_{n}(t)$, we obtain

$$
\begin{aligned}
& \frac{1}{2} \frac{d}{d t} \int_{\Omega}\left|u_{n}(x, t)\right|^{2} d x+M\left(\left[u_{n}\right]_{s, p}^{p}\right) \iint_{\mathcal{Q}} \frac{\left|u_{n}(x, t)-u_{n}(y, t)\right|^{p}}{|x-y|^{N+s p}} d x d y \\
& \quad+\int_{\Omega} f\left(x, u_{n}\right) u_{n} d x=\int_{\Omega} g(x) u_{n} d x .
\end{aligned}
$$

Let $u_{0} \in W_{0} \cap L^{q}(\Omega)$. Then, multiplying (3.1) by $\eta_{n}^{\prime}(t)$, we get

$$
\int_{\Omega}\left|\frac{\partial u_{n}(x, t)}{\partial t}\right|^{2} d x+\frac{d}{d t}\left[\mathscr{M}\left(\left[u_{n}\right]_{s, p}^{p}\right)+\int_{\Omega} F\left(x, u_{n}\right) d x\right]=\int_{\Omega} g(x) \frac{\partial u_{n}(x, t)}{\partial t} d x .
$$

It follows from $\left(M_{1}\right),(1.3),(3.2)$, and the Hölder inequality that

$$
\frac{1}{2} \frac{d}{d t} \int_{\Omega}\left|u_{n}(x, t)\right|^{2} d x+C_{1} \int_{\Omega}\left|u_{n}(x, t)\right|^{q} d x \leq C|\Omega|+\|g(x)\|_{2}\left\|u_{n}(x, t)\right\|_{2} .
$$

Further, by $q \geq 2$ and the Young inequality, we obtain

$$
\frac{1}{2} \frac{d}{d t} \int_{\Omega}\left|u_{n}(x, t)\right|^{2} d x+\frac{C_{1}}{2} \int_{\Omega}\left|u_{n}(x, t)\right|^{2} d x \leq\left[\left(C+C_{1}\right)|\Omega|+\frac{2}{C_{1}}\|g(x)\|_{2}^{2}\right],
$$

which implies that

$$
\begin{aligned}
& \int_{\Omega}\left|u_{n}(x, t)\right|^{2} d x \\
& \quad \leq \int_{\Omega}\left|u_{0 n}(x, 0)\right|^{2} d x e^{-C_{1} t}+\frac{2\left(C+C_{1}\right)|\Omega|}{c_{1}}\left(1-e^{-C_{1} t}\right)+\frac{4}{C_{1}} \int_{0}^{t}\|g\|_{2}^{2} e^{C_{1}(\tau-t)} d \tau \\
& \quad \leq C
\end{aligned}
$$

where $C>0$ denotes various constants independent of $n$ and $t$. This together with (3.2) deduces that the local solution $u_{n}$ can be extended to $(0, \infty)$.

Then, using a similar discussion as that in [21], we can obtain that the limit of $\left\{u_{n}\right\}$ is a solution of problem (1.1).

Next we prove that problem (1.1) only has one solution. Assume that $u$ and $v$ are two solutions of problem (1.1). Taking $\varphi=u-v$ as a test function in Definition 1.1, we have

$$
\begin{aligned}
& \frac{1}{2} \frac{d}{d t} \int_{\Omega}|u-v|^{2} d x+M\left([u]_{s, p}^{p}\right)\langle u, u-v\rangle_{W_{0}}-M\left([v]_{s, p}^{p}\right)\langle u, u-v\rangle_{W_{0}} \\
& \quad+\int_{\Omega}(f(x, u)-f(x, v))(u-v) d x=0 .
\end{aligned}
$$


Note that

$$
\langle u, u-v\rangle_{W_{0}}=[u]_{s, p}^{p}-\langle u, v\rangle_{W_{0}} .
$$

By the Young inequality, we have

$$
\begin{aligned}
\langle u, v\rangle_{W_{0}} & =\iint_{\mathcal{Q}} \frac{|u(x)-u(y)|^{p-2}(u(x)-u(y))(v(x)-v(y))}{|x-y|^{N+s p}} d x d y \\
& \leq\left(1-\frac{1}{p}\right)[u]_{s, p}^{p}+\frac{1}{p}[v]_{s, p}^{p} .
\end{aligned}
$$

Thus,

$$
\langle u, u-v\rangle_{W_{0}} \geq \frac{1}{p}\left([u]_{s, p}^{p}-[v]_{s, p}^{p}\right)
$$

Similarly,

$$
\langle v, u-v\rangle_{W_{0}} \leq \frac{1}{p}\left([u]_{s, p}^{p}-[v]_{s, p}^{p}\right)
$$

Using the above inequalities and assumption (1.2), we arrive at the inequality

$$
\frac{1}{2} \frac{d}{d t} \int_{\Omega}|u-v|^{2} d x+\frac{1}{p}\left[M\left([u]_{s, p}^{p}\right)-M\left([v]_{s, p}^{p}\right)\right]\left([u]_{s, p}^{p}-[v]_{s, p}^{p}\right) \leq \lambda \int_{\Omega}|u-v|^{2} d x
$$

Since $M$ is a nondecreasing function, we deduce that

$$
\frac{d}{d t} \int_{\Omega}|u-v|^{2} d x \leq 2 \lambda \int_{\Omega}|u-v|^{2} d x
$$

which implies that $u-v=0$ a.e. in $\Omega \times(0, \infty)$. Hence the solution is unique. With a similar discussion as the uniqueness of solution, we can obtain the continuity of the mapping $u_{0} \rightarrow u(t)$ in $L^{2}(\Omega)$.

Now we define a functional $E: W_{0} \rightarrow \mathbb{R}$ by

$$
E(u)=\frac{1}{p} \mathscr{M}\left([u]_{s, p}^{p}\right)+\int_{\Omega} F(x, u) d x-\int_{\Omega} g(x) u d x
$$

for all $u \in W_{0}$, where $F(x, u)=\int_{0}^{u} f(x, \xi) d \xi$. Then we have the following.

Lemma 3.1 Assume that $u_{0} \in W_{0} \cap L^{q}(\Omega)$. Let $u$ be a solution of problem (1.1), then

$$
E(u(x, t))=E\left(u_{0}\right)-\int_{0}^{t} \int_{\Omega}\left|u_{\tau}(x, \tau)\right|^{2} d x d \tau, \quad t>0 .
$$

Proof Let us recall that the solution of problem (1.1) can be obtained as the limit of the following sequence of Galerkin's approximation (see [25]):

$$
u_{n}(x, t)=\sum_{j=1}^{n}\left(g_{n}(t)\right)_{j} e_{j}(x), \quad n=1,2, \ldots
$$


where $g_{n}(t) \in C^{1}[0, T]$ and $\left\{e_{j}\right\} \subset C_{0}^{\infty}(\Omega)$ is an orthonormal basis in $L^{2}(\Omega)$. Let $u$ be a sufficiently smooth solution of problem (1.1)(or the approximate solution $u_{n}$ ). Choosing $\varphi=u_{t}$ in Definition 1.1 and using the fact that

$$
\left\langle u, u_{t}\right\rangle_{W_{0}}=\frac{1}{p} \frac{d}{d t} \mathscr{M}\left([u]_{s, p}^{p}\right)
$$

we have

$$
\int_{\Omega}\left|u_{t}\right|^{2} d x+\frac{d}{d t} E(u(x, t))=0
$$

which implies that the function $E(u(x, t))$ is nonincreasing with respect to $t$. Moreover, integrating the above equality with respect to $t$ from 0 to $t$, we arrive at the equality

$$
\int_{0}^{t} \int_{\Omega}\left|u_{\tau}\right|^{2} d x d \tau+E(u(x, t))-E\left(u_{0}\right)=0 .
$$

This ends the proof.

By Theorem 3.1, the solution of problem (1.1) generates a semigroup $\{S(t)\}_{t \geq 0}$ in $L^{2}(\Omega)$. Next, we show that the semigroup possesses a global attractor in $L^{q}(\Omega)$.

Theorem 3.2 Under the assumptions of Theorem 3.1, the semigroup $\{S(t)\}_{t \geq 0}$ associated with problem (1.1) possesses an absorbing set in $L^{2}(\Omega)$ and $W_{0} \cap L^{q}(\Omega)$, respectively.

Proof Taking $\varphi=u$ in Definition 1.1, we obtain

$$
\frac{1}{2} \frac{d}{d t} \int_{\Omega}|u|^{2} d x+M\left([u]_{s, p}^{p}\right)[u]_{s, p}^{p}+\int_{\Omega} f(x, u) u d x=\int_{\Omega} g u d x .
$$

Note that assumption (1.3) implies that

$$
\int_{\Omega} f(x, u) u d x \geq c_{1} \int_{\Omega}|u|^{q} d x-c|\Omega| .
$$

This together with the Young inequality and assumption $\left(M_{1}\right)$ yields that

$$
\frac{1}{2} \frac{d}{d t} \int_{\Omega}|u|^{2} d x+m_{0}[u]_{s, p}^{p}+c_{1} \int_{\Omega}|u|^{q} d x \leq C_{\varepsilon} \int_{\Omega}|g|^{2} d x+\varepsilon \int_{\Omega}|u|^{2} d x+c|\Omega| .
$$

Using the Young inequality, one can deduce that

$$
\begin{aligned}
\int_{\Omega}|u|^{2} d x & \leq \int_{\Omega} \frac{2}{q}|u|^{q} d x+\int_{\Omega} \frac{q-2}{q} d x \\
& =\frac{2}{q} \int_{\Omega}|u|^{q} d x+\frac{q-2}{q}|\Omega| .
\end{aligned}
$$

Thus,

$$
\frac{q}{2} c_{1} \int_{\Omega}|u|^{2} d x \leq c_{1} \int_{\Omega}|u|^{q} d x+\frac{q-1}{2} c_{1}|\Omega| .
$$


Inserting this inequality into (3.6), we get

$$
\begin{aligned}
& \frac{1}{2} \frac{d}{d t} \int_{\Omega}|u|^{2} d x+m_{0}[u]_{s, p}^{p}+\frac{q}{2} c_{1} \int_{\Omega}|u|^{2} d x \\
& \quad \leq \varepsilon^{-1} \int_{\Omega}|g|^{2} d x+\varepsilon \int_{\Omega}|u|^{2} d x+\left(c+\frac{q-1}{2} c_{1}\right)|\Omega| .
\end{aligned}
$$

Choose $\varepsilon=\frac{q c_{1}}{4}$. Then

$$
\begin{aligned}
& \frac{1}{2} \frac{d}{d t} \int_{\Omega}|u|^{2} d x+\frac{q c_{1}}{4} \int_{\Omega}|u|^{2} d x \\
& \quad \leq \frac{4}{q c_{1}} \int_{\Omega}|g|^{2} d x+\left(c+\frac{q-1}{2} c_{1}\right)|\Omega| .
\end{aligned}
$$

Then, using a similar discussion as (3.4), we get that there exists $t_{0}>0$ such that

$$
\|u(x, t)\|_{2} \leq C \quad \text { for any } t \geq t_{0}
$$

Thus, the semigroup has an absorbing set in $L^{2}(\Omega)$. Integrating (3.6) with respect to $t$ over $[t, t+1], t \geq t_{0}$, we obtain

$$
\begin{aligned}
& \int_{t}^{t+1}\left(m_{0}[u(x, \tau)]_{s, p}^{p}+c_{1}\|u(x, \tau)\|_{q}^{q}\right) d \tau \\
& \quad \leq C_{\varepsilon}\|g\|_{2}^{2}+\|u(x, t)\|_{2}^{2}+C|\Omega| \leq C \quad \text { for } t \geq t_{0}
\end{aligned}
$$

which implies that

$$
\int_{t}^{t+1}\left([u(x, \tau)]_{s, p}^{p}+\|u(x, \tau)\|_{q}^{q}\right) d \tau \leq C \quad \text { for } t \geq t_{0} .
$$

On the other hand, using Lemma 3.1 and the Young inequality, we deduce

$$
\frac{1}{2} \int_{\Omega}\left|u_{t}\right|^{2} d x+\frac{d}{d t} \mathscr{M}\left([u]_{s, p}^{p}\right)+\frac{d}{d t} \int_{\Omega} F(x, u) d x \leq \frac{1}{2}\|g\|_{2}^{2} .
$$

By assumption (1.3), we have

$$
c_{1}|u|^{q}-c \leq F(x, u) \leq c_{2}|u|^{q}+c .
$$

Integrating (3.9) over $[\tau, t+1], t_{0} \leq t<\tau<t+1$, one can deduce

$$
\begin{aligned}
& \mathscr{M}\left([u(x, t)]_{s, p}^{p}\right)+\int_{\Omega} F(x, u(x, t)) d x \\
& \quad \leq C\|g\|_{2}^{2}+\left(\mathscr{M}\left([u(x, \tau)]_{s, p}^{p}\right)+\int_{\Omega} F(x, u(x, \tau)) d x\right) .
\end{aligned}
$$


Integrating the above inequality with respect to $\tau$ between $t$ and $t+1$, we obtain

$$
\begin{aligned}
& \mathscr{M}\left([u(x, t+1)]_{s, p}^{p}\right)+\int_{\Omega} F(x, u(x, t+1)) d x \\
& \quad \leq C\|g\|_{2}^{2}+\int_{t}^{t+1}\left(\mathscr{M}\left([u(x, \tau)]_{s, p}^{p}\right)+\int_{\Omega} F(x, u(x, \tau)) d x\right) d \tau .
\end{aligned}
$$

Gathering (3.8) and (3.10), we get

$$
[u(x, t)]_{s, p}^{p}+\|u(x, t)\|_{q}^{q} \leq C \quad \text { for all } t \geq t_{0}+1 .
$$

The proof is complete.

By the compact imbedding results in [35] and [5, Theorem 6.7], we are now in a position to obtain the global attractor in $L^{q}(\Omega)$.

Proof of Theorem 1.1 The proof is inspired by [30]. Let $B_{0}$ be an absorbing set in $L^{q}(\Omega)$. We define the $\omega$-limit set of $B_{0}$ as

$$
\omega\left(B_{0}\right):=\bigcap_{\tau \geq 0} \widehat{\bigcup}_{t \geq \tau} S(t) B_{0}{ }^{L^{q}(\Omega)} .
$$

Here $A^{L^{q}(\Omega)}$ denotes the closure of $A$ in the topology of $L^{q}(\Omega)$. Note that $\varphi \in \omega\left(B_{0}\right)$ if and only if there exist sequences $\left\{\varphi_{n}\right\} \subset B_{0}$ and $t_{n} \rightarrow \infty$ such that

$$
S\left(t_{n}\right) \varphi_{n} \rightarrow \varphi \quad \text { as } n \rightarrow \infty .
$$

Set $\mathscr{A}=\omega\left(B_{0}\right)$. Next we verify that $\mathscr{A}$ is a global attractor of the semigroup $S(t)$ in $L^{q}(\Omega)$.

(1) $\mathscr{A}$ is compact. Clearly, by the compact imbedding results in [35] and [5, Theorem 6.7], one can obtain that $\mathscr{A}$ is compact in $L^{q}(\Omega)$ being $q \in(2, N p /(N-s p))$.

(2) $\mathscr{A}$ is invariant. If $v \in S(t) \mathscr{A}$, then $v=S(t) \varphi, \varphi \in \mathscr{A}$. Thus, there exist $\varphi_{n}$ and $t_{n}$ such that

$$
S(t) S\left(t_{n}\right) \varphi_{n}=S\left(t+t_{n}\right) \varphi_{n} \rightarrow S(t) \varphi=v
$$

which implies that $v \in \mathscr{A}$. If $v \in \mathscr{A}$, then there exist $\varphi_{n} \in B_{0}$ and $t_{n} \rightarrow \infty$ such that $S\left(t_{n}\right) \varphi_{n} \rightarrow v$. Observe that, for $t_{n} \geq t$, the sequence $S\left(t_{n}-t\right) \varphi_{n}$ is compact in $L^{q}(\Omega)$. Thus, there exist a subsequence $t_{n_{k}} \rightarrow \infty$ and $\varphi \in L^{q}(\Omega)$ such that $S\left(t_{n_{k}}-t\right) \varphi_{n_{k}} \rightarrow \varphi$. It follows that $\varphi \in \mathscr{A}$. By the continuity of $S(t)$, we deduce

$$
S\left(t_{n_{k}}\right) \varphi_{n_{k}}=S(t) S\left(t_{n_{k}}-t\right) \varphi_{n_{k}} \rightarrow S(t) \varphi=v .
$$

It yields that $v \in S(t) \mathscr{A}$. Consequently, we obtain that $S(t) \mathscr{A}=\mathscr{A}$.

(3) $\mathscr{A}$ attracts any bounded sets in $L^{q}(\Omega)$. Arguing by contradiction, we assume that for some bounded set $B_{1}$ of $L^{2}(\Omega)$, $\operatorname{dist}\left(S(t) B_{1}, \mathscr{A}\right)$ does not limit to 0 as $t \rightarrow \infty$. Hence there exist $\delta>0$ and a sequence $t_{n} \rightarrow \infty$ such that, for all $n$,

$$
\operatorname{dist}\left(S\left(t_{n}\right) B_{1}, \mathscr{A}\right) \geq \delta>0 .
$$


For each $n \geq 1$, there exists $\varphi_{n} \in B_{1}$ such that

$$
\operatorname{dist}\left(S\left(t_{n}\right) \varphi_{n}, \mathscr{A}\right) \geq \frac{\delta}{2}>0
$$

Recall that $B_{0}$ is an absorbing set. Then $S\left(t_{n}\right) \varphi_{n} \subset B_{0}$ for $t_{n} \geq t_{0}:=t_{0}(B)$. Since $S\left(t_{n}\right) \varphi_{n}$ is compact, there exist $\varphi \in L^{q}(\Omega)$ and a subsequence of $t_{n}$ denoted by $t_{n_{k}}$ such that

$$
\varphi=\lim _{n_{k} \rightarrow \infty} S\left(t_{n_{k}}\right) \varphi_{n_{k}}=\lim _{n_{k} \rightarrow \infty} S\left(t_{n_{k}}-t_{0}\right) S\left(t_{0}\right) \varphi_{n_{k}}
$$

It follows from $S\left(t_{0}\right) \varphi_{n} \in B_{0}$ that $\varphi \in \mathscr{A}_{q}$, which contradicts (3.11).

\section{Infinite dimensional global attractors}

In this section, we study the fractal dimension of the global attractor. First, we prove that the $Z_{2}$ index of the global attractor is infinite. Then, by the Mané projection theorem [18], we obtain the infinite dimensionality of the global attractor.

Let $X$ be a Banach space. Denote by $\sum=\{A \subset V: A$ is closed, $A=-A\}$ the class of closed symmetric subsets of $X$. For any $A \in \sum$, the $Z_{2}$ index of $A$ is defined as follows:

$$
\gamma(A)=\left\{\begin{array}{l}
\inf \left\{m: \exists h \in C^{0}\left(A ; \mathbb{R}^{m} \backslash\{0\}\right), h(-u)=-h(u)\right\} \\
\infty \quad \text { if }\{\cdots\}=\emptyset, \text { in particular if } 0 \in A ; \\
0 \quad A=\emptyset
\end{array}\right.
$$

Now we list some properties of $Z_{2}$ which will be used later, for more details see [29].

Lemma 4.1 The $Z_{2}$ index defined on $\Sigma$ satisfies the following properties:

(1) $\gamma(A)=0 \Leftrightarrow A=\phi$.

(2) If $A \subset B \subset \Sigma$, then $\gamma(A) \leq \gamma(B)$.

(3) For any $A, B \subset \Sigma, \gamma(A \cup B) \leq \gamma(A)+\gamma(B)$.

(4) If $A \in \Sigma$ is a compact set, then $\exists \delta>0$ such that $\gamma\left(\overline{U_{\delta}(A)}\right)=\gamma(A)$, where $U_{\delta}(A)$ is a symmetric $\delta$-neighborhood of $A$.

(5) $\gamma(A) \leq \gamma(\overline{h(A)}), \forall A \in \Sigma$, and $h: V \rightarrow V$ is an odd and continuous function.

To prove Theorem 1.2, we need the following lemma.

Lemma 4.2 Let $\{S(t)\}_{t \geq 0}$ be an odd semigroup on a complete metric space $X$, which possesses a symmetric global attractor $\mathscr{A}$. Then, for any $m \in \mathbb{N}$, there exists a neighborhood $U(0)$ of 0 such that the $Z_{2}$ index of the set $\mathscr{A} \backslash U(0)$ satisfies $\gamma(\mathscr{A} \backslash U(0)) \geq m$.

Proof We first show that, for any integer $m>0$, there exists a symmetric set $B_{m}$ such that

$$
\gamma\left(B_{m}\right) \geq m \quad \text { and } \quad \omega\left(B_{m}\right)=\bigcap_{\tau \geq 0} \overline{\bigcup_{t \geq \tau} S(t) B_{m}} \subset \mathscr{A} \backslash\{0\}
$$

For any $k \in \mathbb{N}^{+}$, let $V_{k}$ be a $k$-dimensional subspace of $W_{0} \cap L^{q}(\Omega)$. Set $A_{k}=\left\{u \in V_{k}:[u]_{s, p}=\right.$ $1\}$, then $A_{k}$ is compact in $W_{0} \cap L^{q}(\Omega)$ and $L^{r}(\Omega)$. Since all norms are equivalent on a finite dimensional Banach space, there exists $C>0$ such that

$$
\|u\|_{r} \geq C[u]_{s, p}=C \quad \text { for all } u \in A_{m} .
$$


Set $\epsilon A_{m}=\left\{\epsilon u: u \in A_{m}\right\}, 0<\epsilon<1$. Then $\gamma\left(\epsilon A_{m}\right)=\gamma\left(A_{m}\right)=m$. For $v=\epsilon u \in \epsilon A_{m}$, we have

$$
\begin{aligned}
E(\nu) & =\frac{1}{p} \mathscr{M}\left(\epsilon^{p}[u]_{s, p}^{p}\right)+\epsilon^{q} \frac{1}{q} \int_{\Omega}|u|^{q} d x-\epsilon^{r} \int_{\Omega}|u|^{r} d x \\
& \leq \frac{1}{p} \epsilon^{p} \max _{\tau \in[0,1]} M(\tau)+C \epsilon^{q}-C \epsilon^{r} .
\end{aligned}
$$

Since $2 \leq r<p$ and $r<q$, for $\epsilon$ small enough such that $E(v)<0$ for all $v \in \epsilon A_{m}$. Now fix $\epsilon>0$ such that $E(\nu)<0$. Since $E(0)=0$ and the function $t \rightarrow E(u(t))$ is nonincreasing, we have $\omega\left(\epsilon A_{m}\right) \subset \mathscr{A} \backslash\{0\}$. Thus, (4.1) holds true by taking $B_{m}=\varepsilon A_{m}$.

Since $B_{m} \subset \mathscr{A} \backslash\{0\}$ and $B_{m}$ is closed and compact, there exist open neighborhoods of 0 and $\omega(B)$, denoted respectively by $U(0)$ and $\mathscr{N}\left(B_{m}\right)$, such that

$$
U(0) \cap \mathscr{N}\left(B_{m}\right)=\emptyset .
$$

Since $S(t) B_{m} \subset \mathscr{N}\left(B_{m}\right)$ for $t$ large enough, we have $S(t) B_{m} \subset \mathscr{N}(\mathscr{A})$ for $t$ large enough. Therefore, there exists $T$ such that, for $t>T$,

$$
S(t) B_{m} \subset \mathscr{N}\left(B_{m}\right) \subset \mathscr{N}(\mathscr{A}) \backslash U(0) \subset \mathscr{N}(\mathscr{A} \backslash U(0))
$$

Note that $\mathscr{A} \backslash U(0)$ is compact. Choosing a proper neighborhood $\mathscr{N}(\mathscr{A} \backslash U(0))$, by (4) in Lemma 4.1, we have

$$
\gamma(\mathscr{A} \backslash U(0))=\gamma(\overline{\mathscr{N}(\omega(A) \backslash U(0))}) \geq \gamma\left(\overline{S(t) B_{m}}\right) \geq \gamma\left(B_{m}\right) \geq m, \quad t \text { large enough. }
$$

The proof is complete.

Proof of Theorem 1.2 By our assumptions, one can obtain that the semigroup of problem (1.9) is odd. Indeed, for any $u_{0} \in L^{2}(\Omega)$, clearly, $-u_{0} \in L^{2}(\Omega)$. Let $u$ be the unique solution of problem (1.9) with initial data $u_{0}$. Since $f$ is odd, $-u$ is the unique solution of problem (1.9) corresponding to initial data $-u_{0}$. Thus, $S(t)\left(-u_{0}\right)=-u=-S(t) u_{0}$, i.e., $S(t)$ is odd.

Let $B$ be a symmetric absorbing set. Then the symmetry of the global attractor follows from the fact that

$$
\mathscr{A}=\omega(B)=\bigcap_{s \geq 0} \overline{\bigcup_{t \geq s} S(t) B} .
$$

Finally, we may take a linear (and thus odd) projection in the Mané projection theorem (see [18]). If there exists $m \in \mathbb{N}$ such that the fractal dimension of $\mathscr{A}$ is less than $m$, then every symmetric closed subset of the attractor (not containing zero) has a $Z_{2}$ index less than $2 m+1$, which contradicts Lemma 4.2. Thus the fractal dimension of the global attractor is infinite. 
Availability of data and materials

Data sharing not applicable to this paper as no datasets were generated or analyzed during the current study.

\section{Competing interests}

The authors declare that they have no competing interests.

\section{Authors' contributions}

All authors read and approved the final manuscript.

\section{Publisher's Note}

Springer Nature remains neutral with regard to jurisdictional claims in published maps and institutional affiliations.

\section{Received: 2 July 2020 Accepted: 2 January 2021 Published online: 21 January 2021}

\section{References}

1. Barrios, B., Colorado, E., de Pablo, A., Sánchez, U.: On some critical problems for the fractional Laplacian operator. J. Differ. Equ. 252, 6133-6162 (2012)

2. Caffarelli, L:: Some nonlinear problems involving non-local diffusions. In: ICIAM 07-6th International Congress on Industrial and Applied Mathematics, pp. 43-56. Eur. Math. Soc., Zürich (2009)

3. Caffarelli, L.: Non-local diffusions, drifts and games. In: Nonlinear Partial Differential Equations, Abel Symposia, vol. 7, pp. 37-52 (2012)

4. Chueshov, I., Lasiecka, I.: Long-Time Behavior of Second Order Evolution Equations with Nonlinear Damping. Memoirs of AMS, vol. 912. Am. Math. Soc., Providence (2008)

5. Di Nezza, E., Palatucci, G., Valdinaci, E.: Hitchhiker's guide to the fractional Sobolev spaces. Bull. Sci. Math. 136, 521-573 (2012)

6. Efendiev, M.A., Ôtani, M.: Infinite-dimensional attractors for evolution equations with $p$-Laplacian and their Kolmogorov entropy. Differ. Integral Equ. 20, 1201-1209 (2007)

7. Fife, P.: Some nonclassical trends in parabolic and parabolic-like evolutions. In: Trends in Nonlinear Analysis, pp. 153-191. Springer, Berlin (2003)

8. Fiscella, A., Servadei, R., Valdinoci, E.: Density properties for fractional Sobolev spaces. Ann. Acad. Sci. Fenn., Math. 40 235-253 (2015)

9. Fiscella, A., Valdinoci, E.: A critical Kirchhoff type problem involving a nonlocal operator. Nonlinear Anal. 94, 156-170 (2014) (2015) 235-253

10. Geredeli, P.G.: On the existence of regular global attractor for $p$-Laplacian evolution equation. Appl. Math. Optim. 71 517-532 (2015)

11. Hartman, P.: Ordinary Differential Equations, 2nd edn. Birkhäuser, Boston (1982)

12. Hurtado, E.J.: Non-local diffusion equations involving the fractional $p(\cdot)$-Laplacian. J. Dyn. Differ. Equ. 32, 557-587 (2020)

13. Iannizzotto, A., Liu, S., Perera, K., Squassina, M.: Existence results for fractional $p$-Laplacian problems via Morse theory. Adv. Calc. Var. 9, 101-126 (2016)

14. Kirchhoff, G.: Vorlesungen über Mathematische Physik. Mechanik, Teubner, Leipzig (1883)

15. Laskin, N.: Fractional quantum mechanics and Lévy path integrals. Phys. Lett. A 268, 298-305 (2000)

16. Lindgren, E., Lindqvist, P.: Fractional eigenvalues. Calc. Var. Partial Differ. Equ. 49, 795-826 (2014)

17. Lorenzo, B., Enea, P.: The second eigenvalue of the fractional p-Laplacian. Adv. Calc. Var. 9, 323-356 (2016)

18. Mané, R.: Lecture Notes in Math., vol. 898, pp. 230-242, Springer, New York (1981)

19. Mingqi, X., Rădulescu, V., Zhang, B.: Fractional Kirchhoff problems with critical Trudinger-Moser nonlinearity. Calc. Var. Partial Differ. Equ. 58, 57 (2019)

20. Mingqi, X., Rădulescu, V.D., Zhang, B.: Nonlocal Kirchhoff problems with singular exponential nonlinearity. Appl. Math. Optim. (2020). https://doi.org/10.1007/s00245-020-09666-3

21. Mingqi, X., Radulescu, V.D., Zhang, B.L.: Nonlocal Kirchhoff diffusion problems: local existence and blow-up of solutions. Nonlinearity 31, 3228-3250 (2018)

22. Molica Bisci, G., Rădulescu, V:: Ground state solutions of scalar field fractional for Schrödinger equations. Calc. Var. Partial Differ. Equ. 54, 2985-3008 (2015)

23. Niu, W.: Long-time behavior for a nonlinear parabolic problem with variable exponents. J. Math. Anal. Appl. 393 $56-65(2012)$

24. Niu, W., Zhong, C.: Global attractors for the $p$-Laplacian equations with nonregular data. J. Math. Anal. Appl. 392 123-135 (2012)

25. Pan, N., Zhang, B., Cao, J.: Degenerate Kirchhoff-type diffusion problems involving the fractional $p$-Laplacian. Nonlinear Anal., Real World Appl. 37, 56-70 (2017)

26. Pucci, P., Xiang, M.Q., Zhang, B.L.: Multiple solutions for nonhomogeneous Schrödinger-Kirchhoff type equations involving the fractional $p$-Laplacian in $\mathbb{R}^{N}$. Calc. Var. Partial Differ. Equ. 54, 2785-2806 (2015)

27. Pucci, P., Xiang, M.Q., Zhang, B.L.: A diffusion problem of Kirchhoff type involving the nonlocal fractional $p$-Laplacian. Discrete Contin. Dyn. Syst. 37, 4035-4051 (2017)

28. Servadei, R., Valdinoci, E.: The Brezis-Nirenberg result for the fractional Laplacian. Trans. Am. Math. Soc. 367, 67-102 (2015)

29. Struwe, M.: Variational Methods, Applications to Nonlinear Partial Differential Equations and Hamiltonian Systems, 2nd edn. Springer, Berlin (1996)

30. Temam, R.: Infinite-Dimensional Dynamical Systems in Mechanics and Physics. Springer, New York (1997)

31. Vázquez, J.L.: Nonlinear diffusion with fractional Laplacian operators. In: Nonlinear Partial Differential Equations, Abel Symp., vol. 7, pp. 271-298. Springer, Heidelberg (2012) 
32. Wang, M., Huang, J.H.: Finite dimensionality of the global attractor for a fractional Schrödinger equation on $\mathbb{R}$. Appl. Math. Lett. 98, 432-437 (2019)

33. Xiang, M.Q., Pucci, P., Squassina, M., Zhang, B.L.: Nonlocal Schrödinger-Kirchhoff equations with external magnetic field. Discrete Contin. Dyn. Syst. 37, 1631-1649 (2017)

34. Xiang, M.Q., Yang, D.: Nonlocal Kirchhoff problems: extinction and non-extinction of solutions. J. Math. Anal. Appl. 477, 133-152 (2019)

35. Xiang, M.Q., Zhang, B.L., Ferrara, M.: Existence of solutions for Kirchhoff type problem involving the non-local fractional $p$-Laplacian. J. Math. Anal. Appl. 424, 1021-1041 (2015)

36. Xiang, M.Q., Zhang, B.L., Rădulescu, V.D.: Existence of solutions for a bi-nonlocal fractional p-Kirchhoff type problem. Comput. Math. Appl. 71, 255-266 (2016)

37. Yang, M., Sun, C., Zhong, C.: Global attractors for p-Laplacian equation. J. Math. Anal. Appl. 327, 1130-1142 (2007)

38. Zhong, $C ., N i u, W$.: On the $Z_{2}$ index of the global attractor for a class of $p$-Laplacian equations. Nonlinear Anal. 73 , 3698-3704 (2010)

Submit your manuscript to a SpringerOpen ${ }^{\circ}$ journal and benefit from:

- Convenient online submission

- Rigorous peer review

- Open access: articles freely available online

- High visibility within the field

- Retaining the copyright to your article

Submit your next manuscript at $\boldsymbol{\nabla}$ springeropen.com 